\title{
Constraint propagation in the family of ADM systems
}

\author{
Gen Yoneda ${ }^{\dagger}$ and Hisa-aki Shinkai $\ddagger$ \\ yoneda@mse.waseda.ac.jp shinkai@gravity.phys.psu.edu \\ $\dagger$ Department of Mathematical Sciences, Waseda University, Shinjuku, Tokyo, 169-8555, Japan \\ ¥ Centre for Gravitational Physics and Geometry, 104 Davey Lab., Department of Physics, \\ The Pennsylvania State University, University Park, Pennsylvania 16802-6300, USA
}

(March 8, 2001, gr-qc/0103032, CGPG-01/3-2)

\begin{abstract}
The current important issue in numerical relativity is to determine which formulation of the Einstein equations provides us with stable and accurate simulations. Based on our previous work on "asymptotically constrained" systems, we here present constraint propagation equations and their eigenvalues for the Arnowitt-Deser-Misner (ADM) evolution equations with additional constraint terms (adjusted terms) on the right hand side. We conjecture that the system is robust against violation of constraints if the amplification factors (eigenvalues of Fourier-component of the constraint propagation equations) are negative or pure-imaginary. We show such a system can be obtained by choosing multipliers of adjusted terms. Our discussion covers Detweiler's proposal (1987) and Frittelli's analysis (1997), and we also mention the so-called conformal-traceless ADM systems.
\end{abstract}

PACS numbers: 04.20.-q, 04.20.Fy, 04.25.-g, 04.25.Dm

\section{INTRODUCTION}

The effort to solve the Einstein equations numerically - so-called Numerical Relativity - is now providing an interesting bridge between mathematical relativists and numerical relativists. Most of the simulations have been performed using the Arnowitt-Deser-Misner (ADM) formulation [1] or a modified version. However, the ADM formulation has not been proven to be a well-posed system, since its evolution equations do not present a hyperbolic form in its original/standard formulation.

Most simulations are performed using the "free evolution" procedures: (1) solve the Hamiltonian and momentum constraints to prepare the initial data, (2) integrate the evolution equations by fixing gauge conditions, and (3) monitor the accuracy/stability by evaluating the constraints. Many trials have been made in the last few decades, but we have not yet obtained a perfect recipe for long-term stable evolution of the Einstein equations. Here we consider the problem through the form of the equations.

One direction in the community is to rewrite the Einstein evolution equations into a hyperbolic form and to apply it to numerical simulations [2]. This is motivated by the fact that we can prove well-posedness for the evolution of several systems if they have a certain kind of hyperbolic feature. The authors recently derived [3. system of the Einstein equations using Ashtekar's connection variables [5] 1], and compared them numerically [6]. We found that (a) the three levels of hyperbolicity can be obtained by adding constraint terms and/or imposing gauge conditions, (b) there is no drastic difference in the accuracy of numerical evolutions in these three, and (c) the symmetric hyperbolic system is not always the best for reducing numerical errors. Similar results regarding to (a) and (b) are reported by Hern [] based on the Frittelli-Reula formulation [8].

What are, then, the criteria for predicting the stable evolutions of a system? Inspired by the " $\lambda$-system" proposal [9], we have considered a so-called "asymptotically constrained" system, that is, a system robust against the violation of the constraints [10]. The fundamental idea of the " $\lambda$-system" is to introduce artificial flow onto the constraint surface. However, we also found that such a feature can be obtained simply by adding constraint terms to the evolution equations which we named "adjusted systems" 11. We explained the reason why this works by analyzing the evolution equations of the constraints (the propagation of the constraints). We proposed that the stablity of the system can be predicted by analyzing the eigenvalues (amplification factors) of the constraint propagation equations

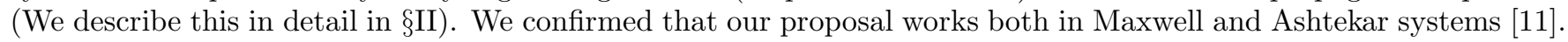

\footnotetext{
${ }^{1}$ We derived weakly, strongly (= diagonalizable) and symmetric hyperbolic systems. The mathematical inclusion relation is weakly hyperbolic $\ni$ strongly hyperbolic $\ni$ symmetric hyperbolic.
}

See details in 迎. 
The purpose of this article is to apply our proposal to the ADM system(s). Especially, we consider the "adjusting process" (adding constraints in RHS of evolution equations), and the resultant changes to the eigenvalues of the constraint propagation systems. This adjusting process can be seen in many constructions of hyperbolic systems in the references. In fact, the standard $A D M$ for numerical relativists is the version which was introduced by York [12], where the original $A D M$ system [1] has already been adjusted using the Hamiltonian constraint (see more detail in SIII). The advantage of the standard $A D M$ system is reported by Frittelli [13 from the point of the hyperbolicity and the characteristic propagation speed of the constraints. Our discussion extends her analysis to the amplification factors.

One early effort of the adjusting mechanism was presented by Detweiler [14]. Our study also includes his system, and shows that this system actually works as desired for a certain choice of parameter ( $\S[\mathrm{V}$ ). We also study the same procedure for the "conformal-traceless" ADM (CT-ADM) formulations [15.16] which is recently the most popular system in numerical simulations $(\S \bar{V})$.

The analysis in the text is for perturbational violation on a flat background. Further applications are available, but we will discuss them in future reports. In the Appendix, we also give numerical demonstrations of the adjusted-ADM systems discussed in the text.

\section{CONSTRAINT PROPAGATION AND "ADJUSTED SYSTEM"}

We begin by reviewing the background of "adjusted systems" and our conjecture.

The notion of the evolution equations of the constraints is often discussed from the point of whether they form a first class system or not. Fortunately, the constraints in the (original/standard) ADM formulation are known to form a first class system. Due to this fact, numerical relativists only need to monitor the violation of the Hamiltonian and momentum constraints during the free evolution of the initial data.

Our essential idea here is to feed this procedure back into the evolution equations. That is, we adjust the system's evolution equations by characterizing the constraint propagation in advance. Let us describe the procedure in a general form. Suppose we have a set of dynamical variables, $u^{a}\left(x^{i}, t\right)$, and its evolution equations,

$$
\partial_{t} u^{a}=f\left(u^{a}, \partial_{i} u^{a}, \cdots\right),
$$

which should satisfy a set of constraints, $C^{\rho}\left(u^{a}, \partial_{i} u^{a}, \cdots\right) \approx 0$. The evolution equation for $C^{a}$ can be written as

$$
\partial_{t} C^{\rho}=g\left(C^{\rho}, \partial_{i} C^{\rho}, \cdots\right) \text {. }
$$

We can perform two main types of analysis analysis on (2.2):

1. If (2.2) is in a first order form (that is, only includes first-order spatial derivatives), then the level of hyperbolicity and the characteristic speeds (eigenvalues $\lambda^{l}$ of the principal matrix) will definitely deterine the stability of the system. We expect mathematically rigorous well-posed features for strongly or symmetric hyperbolic systems, and the characteristic speeds suggest to us satisfactory criteria for stable evolutions if they are real, and under the propagation speed of the original variables, $u^{a}$, and/or within the causal region of the numerical integration scheme applied.

2. On the other hand, the Fourier transformed-(2.2),

$$
\partial_{t} \hat{C}^{\rho}=\hat{g}\left(\hat{C}^{\rho}\right)
$$

where $C^{\rho}(x, t)=\int \hat{C}^{\rho}(k, t) \exp (i k \cdot x) d^{3} k$, also characterizes the evolution of the constraints independently of its hyperbolicity. As we have proposed and confirmed in [11], the set of eigenvalues $\Lambda^{i}$ of the coefficient matrix in (2.3) provides a kind of amplification factor of the constraint propagation, and predicts the increase/decrease of the violation of the constraints if it exists. More precisely, we showed in [1] that

if the eigenvalues of (2.3) (a) have a negative real-part, or (b) are non-zero (pure-imaginary) eigenvalues, then we see more stable evolutions than a system which does not.

This is because the constraints are damped if the eigenvalues are negative, and are propagating away if the eigenvalues are pure imaginary. We found heuristically that the system becomes more stable (accurate) when as much $\Lambda$ s satisfies the above criteria and/or as large magnitude of $\Lambda$ s away from zeros. (Examples in [11] are of the plane wave propagation in the Maxwell system and the Ashtekar system.) We remark that this eigenvalue analysis requires that we fix a particular background metric for the situation we consider, since the amplification factor depends on the dynamical variables $u^{a}$. 
The above features of the constraint propagation, (2.2), will change when we modify the original evolution equations. Suppose we adjust the RHS of (2.1) by adding the constraints,

$$
\partial_{t} u^{a}=f\left(u^{a}, \partial_{i} u^{a}, \cdots\right)+F\left(C^{\rho}, \partial_{i} C^{\rho}, \cdots\right),
$$

then (2.2) will also be modified as

$$
\partial_{t} C^{\rho}=g\left(C^{\rho}, \partial_{i} C^{\rho}, \cdots\right)+G\left(C^{\rho}, \partial_{i} C^{\rho}, \cdots\right) .
$$

By taking the characteristic speed of (2.5) and the amplification factor of the Fourier transformed-(2.5), the predicted stability of the system (2.4) becomes different to that of the original system, (2.2).

Our proposed "adjusted system" is obtained by finding a certain functional form of $F\left(C^{\rho}, \partial_{i} C^{\rho}, \cdots\right)$ in (2.4) so as to get a more stable prediction in the analysis of the eigenvalues $\lambda^{l}$ and $\Lambda^{i}$. In the following discussion, we show two eigenvalues $\lambda^{l}$ and $\Lambda^{i}$ for each ADM system. We remark again that the term 'characteristic speed' here is not for the dynamical equation (2.1), but for the constraint propagation equations (2.2).

\section{STANDARD ADM SYSTEM}

\section{A. Standard ADM system and its constraint propagation}

We start by analyzing the standard ADM system. By "standard ADM" we mean here the most widely adopted system, due to York [12], with evolution equations

$$
\begin{aligned}
\partial_{t} \gamma_{i j} & =-2 \alpha K_{i j}+\nabla_{i} \beta_{j}+\nabla_{j} \beta_{i}, \\
\partial_{t} K_{i j} & =\alpha R_{i j}^{(3)}+\alpha K K_{i j}-2 \alpha K_{i k} K_{j}^{k}-\nabla_{i} \nabla_{j} \alpha+\left(\nabla_{i} \beta^{k}\right) K_{k j}+\left(\nabla_{j} \beta^{k}\right) K_{k i}+\beta^{k} \nabla_{k} K_{i j},
\end{aligned}
$$

and constraint equations

$$
\begin{aligned}
\mathcal{H} & :=R^{(3)}+K^{2}-K_{i j} K^{i j}, \\
\mathcal{M}_{i} & :=\nabla_{j} K^{j}{ }_{i}-\nabla_{i} K,
\end{aligned}
$$

where $\left(\gamma_{i j}, K_{i j}\right)$ are the induced three-metric and the extrinsic curvature, $\left(\alpha, \beta_{i}\right)$ are the lapse function and the shift covector, $\nabla_{i}$ is the covariant derivative adapted to $\gamma_{i j}$, and $R_{i j}^{(3)}$ is the three-Ricci tensor.

The constraint propagation equations, which are the time evolution equations of the Hamiltonian constraint (3.3) and the momentum constraints (3.4), can be written as

$$
\begin{aligned}
\partial_{t} \mathcal{H} & =\beta^{j}\left(\partial_{j} \mathcal{H}\right)-2 \alpha \gamma^{i j}\left(\partial_{i} \mathcal{M}_{j}\right)+2 \alpha K \mathcal{H}+\alpha\left(\partial_{l} \gamma_{m k}\right)\left(2 \gamma^{m l} \gamma^{k j}-\gamma^{m k} \gamma^{l j}\right) \mathcal{M}_{j}-4 \gamma^{i j}\left(\partial_{j} \alpha\right) \mathcal{M}_{i} \\
\partial_{t} \mathcal{M}_{i} & =-(1 / 2) \alpha\left(\partial_{i} \mathcal{H}\right)+\beta^{j}\left(\partial_{j} \mathcal{M}_{i}\right)+\alpha K \mathcal{M}_{i}-\left(\partial_{i} \alpha\right) \mathcal{H}-\beta^{k} \gamma^{j l}\left(\partial_{i} \gamma_{l k}\right) \mathcal{M}_{j}+\left(\partial_{i} \beta_{k}\right) \gamma^{k j} \mathcal{M}_{j}
\end{aligned}
$$

The simplest derivation of (3.5) and (3.6) is by using the Bianchi identity, which can be seen in Frittelli [13]. (Note that $C$ in 13 is half our $\mathcal{H}$, and we have corrected typos in eq.(11) in $[13])$.

The characteristic part of (3.5) and (3.6) can be extracted as

$$
\partial_{t}\left(\begin{array}{c}
\mathcal{H} \\
\mathcal{M}_{i}
\end{array}\right) \simeq\left(\begin{array}{cc}
\beta^{l} & -2 \alpha \gamma^{i l} \\
-(1 / 2) \alpha \delta_{i}^{l} & \beta^{l} \delta_{i}^{j}
\end{array}\right) \partial_{l}\left(\begin{array}{c}
\mathcal{H} \\
\mathcal{M}_{j}
\end{array}\right)=: P^{l} \partial_{l}\left(\begin{array}{c}
\mathcal{H} \\
\mathcal{M}_{j}
\end{array}\right)
$$

which indicates that the characteristic speeds (eigenvalues of the characteristic matrix, $P^{l}$ ) are

$$
\lambda^{l}=\left(\beta^{l}, \beta^{l}, \beta^{l} \pm \alpha \sqrt{\gamma^{l l}}\right) \quad(\text { no sum over } l) .
$$

Since $\operatorname{rank}\left(P^{l}-\beta^{l}\right)=2$, the matrix $P^{l}$ is diagonalizable, but not the symmetric.

Simply by inserting $(1 / 2)$ in front of $\mathcal{H}$ above, we obtain

$$
\partial_{t}\left(\begin{array}{c}
\mathcal{H} / 2 \\
\mathcal{M}_{i}
\end{array}\right) \simeq\left(\begin{array}{cc}
\beta^{l} & -\alpha \gamma^{i l} \\
-\alpha \delta_{i}^{l} & \beta^{l} \delta_{i}^{j}
\end{array}\right) \partial_{l}\left(\begin{array}{c}
\mathcal{H} / 2 \\
\mathcal{M}_{j}
\end{array}\right)
$$

the characteristic matrix becomes symmetric (with the same eigenvalues). This is a feature of the standard ADM system that was pointed out by Frittelli. (Actually $\mathcal{H} / 2$ is the form originally given by the Lagrangian formulation.) 


\section{B. Amplification factors on the Minkowskii background}

As a first example, we consider the perturbation of the Minkowskii spacetime: $\alpha=1, \beta^{i}=0, \gamma_{i j}=\delta_{i j}$. By taking the linear order contribution, (3.5) and (3.6) are reduced to

$$
\partial_{t}\left(\begin{array}{c}
(1) \hat{\mathcal{H}} \\
(1) \hat{\mathcal{M}}_{i}
\end{array}\right)=\left(\begin{array}{cc}
0 & -2 i k_{j} \\
-(1 / 2) i k_{i} & 0
\end{array}\right)\left(\begin{array}{c}
(1) \hat{\mathcal{H}} \\
(1) \hat{\mathcal{M}}_{j}
\end{array}\right) \text {, }
$$

in Fourier components. The eigenvalues of the coefficient matrix of (3.10), which we call amplification factors, become

$$
\Lambda^{l}=\left(0,0, \pm i \sqrt{k^{2}}\right)
$$

where $k^{2}=k_{x}^{2}+k_{y}^{2}+k_{z}^{2}$. These factors will be compared with others later, but we note that the real parts of all the $\Lambda$ s are zero.

\section{ADJUSTED ADM SYSTEMS}

\section{A. Adjustments}

Generally, we can write the adjustment terms to (3.1) and (3.2) using (3.3) and 3.4) by the following combinations (using up to the first derivative of constraints),

$$
\begin{array}{lll}
\text { adjustment term of } & \partial_{t} \gamma_{i j}: & +P_{i j} \mathcal{H}+Q^{k}{ }_{i j} \mathcal{M}_{k}+p^{k}{ }_{i j}\left(D_{k} \mathcal{H}\right)+q^{k l}{ }_{i j}\left(D_{k} \mathcal{M}_{l}\right), \\
\text { adjustment term of } & \partial_{t} K_{i j}: & +R_{i j} \mathcal{H}+S^{k}{ }_{i j} \mathcal{M}_{k}+r^{k}{ }_{i j}\left(D_{k} \mathcal{H}\right)+s^{k l}{ }_{i j}\left(D_{k} \mathcal{M}_{l}\right),
\end{array}
$$

where $P, Q, R, S$ and $p, q, r, s$ are multipliers (please do not confuse $R_{i j}$ with three Ricci curvature that we write as $\left.R_{i j}^{(3)}\right)$. Since this expression is too general, we mention some restricted cases below.

We remark that our starting system, (3.1) and (3.2), is the standard ADM system for numerical relativists introduced by York 12]. This expression can be obtained from the originally formulated canonical expression by ADM 11, but in that process the Hamiltonian constraint equation is used to eliiminate the three dimensional Ricci scalar. Therefore the standard $A D M$ is already adjusted from the original $A D M$ system. We start our comparison with this point.

\section{B. Original ADM vs Standard ADM}

Frittelli's adjustment analysis [13] can be written in terms of (4.1) and (4.2), as

$$
R_{i j}=(1 / 4)(\mu-1) \alpha \gamma_{i j},
$$

where $\mu$ is a constant and set other multiplier zero. Here $\mu=1$ corresponds to the standard ADM (no adjustment, since $R_{i j}=0$ ), and $\mu=0$ to the original ADM (without any adjustment to the canonical formulation by ADM).

Keeping the multiplier (4.3) in mind, we here discuss the case of non-zero $R_{i j}, S^{k}{ }_{i j}$ (and all other multipliers zero) case. The constraint propagation equations become

$$
\begin{aligned}
\partial_{t} \mathcal{H}= & \beta^{j}\left(\partial_{j} \mathcal{H}\right)-2 \alpha \gamma^{i j}\left(\partial_{i} \mathcal{M}_{j}\right)+2 \alpha K \mathcal{H}+\alpha\left(\partial_{l} \gamma_{m k}\right)\left(2 \gamma^{m l} \gamma^{k j}-\gamma^{m k} \gamma^{l j}\right) \mathcal{M}_{j}-4 \gamma^{i j}\left(\partial_{j} \alpha\right) \mathcal{M}_{i} \\
& +2 K R \mathcal{H}-2 K^{i j} R_{i j} \mathcal{H}+2 K \gamma^{i j} S^{k}{ }_{i j} \mathcal{M}_{k}-2 K^{i j} S^{k}{ }_{i j} \mathcal{M}_{k}, \\
\partial_{t} \mathcal{M}_{i}= & -(1 / 2) \alpha\left(\partial_{i} \mathcal{H}\right)+\beta^{j}\left(\partial_{j} \mathcal{M}_{i}\right)+\alpha K \mathcal{M}_{i}-\left(\partial_{i} \alpha\right) \mathcal{H}-\beta^{k} \gamma^{j l}\left(\partial_{i} \gamma_{l k}\right) \mathcal{M}_{j}+\left(\partial_{i} \beta_{k}\right) \gamma^{k j} \mathcal{M}_{j} \\
& +\gamma^{k j}\left(\partial_{j} R_{k i}\right) \mathcal{H}-\gamma^{j k}\left(\partial_{i} R_{j k}\right) \mathcal{H}+R^{j}{ }_{i}\left(\partial_{j} \mathcal{H}\right)-R_{j k} \gamma^{j k}\left(\partial_{i} \mathcal{H}\right) \\
& +\gamma^{l j}\left(\partial_{j} S^{k}{ }_{l i}\right) \mathcal{M}_{k}-\gamma^{j l}\left(\partial_{i} S^{k}{ }_{j l}\right) \mathcal{M}_{k}+S^{k j}{ }_{i}\left(\partial_{j} \mathcal{M}_{k}\right)-\gamma^{j l} S^{k}{ }_{j l}\left(\partial_{i} \mathcal{M}_{k}\right) \\
& +\left(\partial_{j} \gamma^{k j}\right) R_{k i} \mathcal{H}+\Gamma_{j k}^{j} R^{k}{ }_{i} \mathcal{H}-\Gamma_{j i}^{k} R^{j}{ }_{k} \mathcal{H}-\left(\partial_{i} \gamma^{j k}\right) R_{j k} \mathcal{H} \\
& +\left(\partial_{j} \gamma^{l j}\right) S^{k}{ }_{l i} \mathcal{M}_{k}+\Gamma_{j l}^{j}{ }^{k l}{ }_{i} \mathcal{M}_{k}-\Gamma_{j i}^{l} S^{k j}{ }_{l} \mathcal{M}_{k}-\left(\partial_{i} \gamma^{j l}\right) S^{k}{ }_{j l} \mathcal{M}_{k},
\end{aligned}
$$

that is, (4.4) and (4.5) form a first-order system. The principal part can be written as 


$$
\partial_{t}\left(\begin{array}{c}
\mathcal{H} \\
\mathcal{M}_{i}
\end{array}\right) \simeq\left(\begin{array}{cc}
\beta^{l} & -2 \alpha \gamma^{j l} \\
-(1 / 2) \alpha \delta_{i}^{l}+R^{l}{ }_{i}-\delta_{i}^{l} R_{k m} \gamma^{k m} & \beta^{l} \delta_{i}^{j}+S^{j l}{ }_{i}-\gamma^{m k} \delta_{i}^{l} S^{j}{ }_{m k}
\end{array}\right) \partial_{l}\left(\begin{array}{c}
\mathcal{H} \\
\mathcal{M}_{j}
\end{array}\right) .
$$

The general discussion of the hyperbolicity and characteristic speed of the system (4.6) is hard, so hereafter we restrict ourselves to the case

$$
R_{i j}=\kappa_{1} \alpha \gamma_{i j}, \quad S^{k}{ }_{i j}=\kappa_{2} \beta^{k} \gamma_{i j},
$$

where we recover (4.3) by choosing $\kappa_{1}=(\mu-1) / 4$ and $\kappa_{2}=0$. The eigenvalues of (4.6) then become

$$
\lambda^{l}=\left(\beta^{l}, \beta^{l},\left(1-\kappa_{2}\right) \beta^{l} \pm \sqrt{\alpha^{2} \gamma^{l l}\left(1+4 \kappa_{1}\right)+\left(\kappa_{2} \beta^{l}\right)^{2}}\right) \quad(\text { no sum over } l \text { ) }
$$

and the hyperbolicity of (4.6) can be classified as (i) symmetric hyperbolic when $\kappa_{1}=3 / 2$ and $\kappa_{2}=0$, (ii) strongly hyperbolic when $\alpha^{2} \gamma^{l l}\left(1+4 \kappa_{1}\right)+\kappa_{2}^{2}\left(\beta^{l}\right)^{2}>0$ where $\kappa_{1} \neq-1 / 4$, and (iii) weakly hyperbolic when $\alpha^{2} \gamma^{l l}\left(1+4 \kappa_{1}\right)+$ $\kappa_{2}^{2}\left(\beta^{l}\right)^{2} \geq 0$.

For the case of (4.7) on a Minkowskii background metric, the linear order terms of the constraint propagation equations become

$$
\partial_{l}\left(\begin{array}{c}
(1) \hat{\mathcal{H}} \\
(1) \hat{\mathcal{M}}_{i}
\end{array}\right)=\left(\begin{array}{cc}
0 & -2 i k_{j} \\
-(1 / 2)\left(1+4 \kappa_{1}\right) i k_{i} & 0
\end{array}\right)\left(\begin{array}{c}
(1) \hat{\mathcal{H}} \\
(1) \hat{\mathcal{M}}_{j}
\end{array}\right)
$$

whose Fourier transform gives the eigenvalues

$$
\Lambda^{l}=\left(0,0, \pm \sqrt{-k^{2}\left(1+4 \kappa_{1}\right)}\right) .
$$

That is (two 0s, two pure imaginary) for the standard ADM, and (four 0s) for the original ADM system. Therefore, according to our conjecture, the standard ADM system is expected to have better stability than the original ADM system.

\section{Detweiler's system}

\section{Detweiler's system and its constraint amplification}

Detweiler's modification to ADM [14] can be realized through one choice of the multipliers in (4.1) and (4.2). He found that with a particular combination the evolution of the energy norm of the constraints, $\mathcal{H}^{2}+\mathcal{M}^{2}$, can be negative definite when we apply the maximal slicing condition, $K=0$. (We will comment more on his criteria in \&V C 2.) His adjustment can be written in our notation in (4.1) and 4.2.), as

$$
\begin{aligned}
P_{i j} & =-L \alpha^{3} \gamma_{i j}, \\
R_{i j} & =L \alpha^{3}\left(K_{i j}-(1 / 3) K \gamma_{i j}\right), \\
S^{k}{ }_{i j} & =L \alpha^{2}\left[3\left(\partial_{(i} \alpha\right) \delta_{j)}^{k}-\left(\partial_{l} \alpha\right) \gamma_{i j} \gamma^{k l}\right], \\
s^{k l}{ }_{i j} & =L \alpha^{3}\left[\delta_{(i}^{k} \delta_{j)}^{l}-(1 / 3) \gamma_{i j} \gamma^{k l}\right],
\end{aligned}
$$

everything else zero, where $L$ is a constant. Detweiler's adjustment, (4.12)-(4.14), does not put constraint propagation equation to first order form, so we can not discuss hyperbolicity or the characteristic speed of the constraints.

For the Minkowskii background spacetime, the adjusted constraint propagation equations with above choice of multiplier become

$$
\partial_{l}\left(\begin{array}{c}
(1) \hat{\mathcal{H}} \\
(1) \hat{\mathcal{M}}_{i}
\end{array}\right)=\left(\begin{array}{cc}
-2 L k^{2} & -2 i k_{j} \\
-(1 / 2) i k_{i} & -(L / 2) k^{2} \delta_{i}^{j}-(L / 6) k_{i} k_{j}
\end{array}\right)\left(\begin{array}{c}
(1) \hat{\mathcal{H}} \\
(1) \hat{\mathcal{M}}_{j}
\end{array}\right)
$$

The eigenvalues of the Fourier transform are

$$
\Lambda^{l}=\Lambda^{l}=\left(-(L / 2) k^{2},-(L / 2) k^{2},-(4 L / 3) k^{2} \pm \sqrt{k^{2}\left(-1+(4 / 9) L^{2} k^{2}\right)}\right) .
$$

This indicates negative real eigenvalues if we choose small positive $L$.

We confirmed numerically, using perturbation on Minkowskii, that Detweiler's system presents better accuracy than the standard ADM, but only for small positive L. See the Appendix. 
We comment here on the differences between Detweiler's criteria for stable evolution and ours.

Detweiler calculated the $\mathrm{L} 2$ norm of the constraints, $C_{\rho}$, over the 3 -hypersurface and imposed the negative definiteness of its evolution,

$$
\text { Detweiler's criteria } \Leftrightarrow \partial_{t} \int C_{\rho} C^{\rho} d V<0, \forall \text { non zero } C_{\rho} \text {. }
$$

where $C_{\rho} C^{\rho}=: G^{\rho \sigma} C_{\rho} C_{\sigma}$, and $G_{\rho \sigma}=\operatorname{diag}\left[1, \gamma_{i j}\right]$ for the pair of $C_{\rho}=\left(\mathcal{H}, \mathcal{M}_{i}\right)$.

Assuming the constraint propagation to be $\partial_{t} \hat{C}_{\rho}=A_{\rho}{ }^{\sigma} \hat{C}_{\sigma}$ in the Fourier components, the time derivative of the L2 norm can be written as

$$
\partial_{t}\left(\hat{C}_{\rho} \hat{C}^{\rho}\right)=\left(A^{\rho \sigma}+\bar{A}^{\sigma \rho}+\partial_{t} \bar{G}^{\rho \sigma}\right) \hat{C}_{\rho} \overline{\hat{C}}_{\sigma} .
$$

Together with the fact that the L2 norm is preserved by Fourier transform, we can say, for the case of static background metric,

$$
\text { Detweiler's criteria } \Leftrightarrow \text { eigenvalues of }\left(A+A^{\dagger}\right) \text { are all negative } \forall k \text {. }
$$

On the other hand,

$$
\text { Our criteria } \Leftrightarrow \text { eigenvalues of } A \text { are all negative } \forall k \text {. }
$$

Therefore for the case of static background, Detweiler's criterion is stronger than ours. For example, the matrix

$$
A=\left(\begin{array}{cc}
-1 & a \\
0 & -1
\end{array}\right) \text { where } a \text { is constant }
$$

for the evolution system $\left(\hat{C}_{1}, \hat{C}_{2}\right)$ satisfies our criterion but not Detweiler's when $|a| \geq \sqrt{2}$. This matrix however gives asymptotical decay for $\left(\hat{C}_{1}, \hat{C}_{2}\right)$. Therefore we may say that Detweiler requires the monotonic decay of the constraints, while we assume only asymptotical decay.

We remark that Detweiler's truncations on higher order terms in $C$-norm corresponds to our perturbational analysis; both are based on the idea that the deviations from constraint surface (the errors expressed non-zero constraint value) are initially small.

\section{Another possible adjustment}

\section{Simplified Detweiler system}

Similar to Detweiler's (4.11), we next consider only the adjustment

$$
P_{i j}=\kappa_{0} \alpha \gamma_{i j}
$$

all other multipliers zero in (4.1) and (4.2).

On the Minkowskii background, the Fourier components of the constraint propagation equation can be written as

$$
\partial_{t}\left(\begin{array}{c}
(1) \hat{\mathcal{H}} \\
(1) \hat{\mathcal{M}}_{i}
\end{array}\right)=\left(\begin{array}{cc}
2 \kappa_{0} k^{2} & -2 i k_{j} \\
-(1 / 2) i k_{i} & 0
\end{array}\right)\left(\begin{array}{c}
(1) \hat{\mathcal{H}} \\
(1) \hat{\mathcal{M}}_{j}
\end{array}\right)
$$

and the eigenvalues of the coefficient matrix are

$$
\Lambda^{l}=\left(0,0, \kappa_{0} k^{2} \pm \sqrt{k^{2}\left(-1+\kappa_{0}^{2} k^{2}\right)}\right) .
$$

That is, the amplification factors become $\left(0,0\right.$, two negative reals) for the choice of relatively small negative $\kappa_{0}$.

We also confirmed that this system works as desired. We give a numerical example in the Appendix. 


\section{Adjusting-Hamiltonian-constraints system}

Our final example is a combination of the one in $\S \mathrm{VB}$ and that above, that is

$$
\begin{aligned}
& P_{i j}=\kappa_{0} \alpha \gamma_{i j}, \\
& R_{i j}=\kappa_{1} \alpha \gamma_{i j},
\end{aligned}
$$

all other multipliers zero in (4.1) and (4.2). Similar to the previous one, the Fourier transformed constraint propagation equation is

$$
\partial_{t}\left(\begin{array}{c}
(1) \hat{\mathcal{H}} \\
(1) \hat{\mathcal{M}}_{i}
\end{array}\right)=\left(\begin{array}{cc}
2 \kappa_{0} k^{2} & -2 i k_{i} \\
-(1 / 2) i k_{i}-2 \kappa_{1} i k_{i} & 0
\end{array}\right)\left(\begin{array}{c}
(1) \hat{\mathcal{H}} \\
(1) \hat{\mathcal{M}}_{j}
\end{array}\right)
$$

which gives the eigenvalues

$$
\Lambda^{l}=\left(0,0, \kappa_{0} k^{2} \pm \sqrt{k^{2}\left(-1+\kappa_{0} k^{2}-4 \kappa_{1}\right)}\right) .
$$

We can expect a similar asymptotical stable evolution by choosing $\kappa_{0}$ and $\kappa_{1}$, so as to make the eigenvalues $(0$, 0 , two negative reals).

\section{CONFORMAL-TRACELESS ADM SYSTEMS}

The so-called "conformally decoupled traceless ADM formulation" (CT-ADM) was first developed by the Kyoto group [15]. After the re-discovery that this formulation is more stable than the standard ADM by Baumgarte and Shapiro [16], several groups began to use CT-ADM formulation for their numerical codes, and reported an advantage in stability [17,18]. Along with this conformal decomposition, several hyperbolic formulations have also been proposed 19 21, but they have not yet been applied to numerical simulations.

However, it is not yet clear why CT-ADM gives better stability than ADM. The Potsdam group [22] found that the eigenvalues of CT-ADM evolution equations has fewer "zero eigenvalues" than those of ADM, and they conjectured that the instability can be caused by "zero eigenvalues" that violate "gauge mode". Miller [23] applied von Neumann's stability analysis to the plane wave propagation, and reported that CT-ADM has a wider range of parameters that give us stable evolutions. These studies provide supports of CT-ADM in some sense, but on the other hand, it is also shown that an example of an ill-posed solution in CT-ADM (as well in ADM) [24].

Here, we apply our constraint propagation analysis to this CT-ADM system.

\section{A. CT-ADM equations}

Since one reported feature of CT-ADM is the use of the momentum constraint in RHS of the evolution equations [22], we here present the set of CT-ADM equations carefully for such an replacement of the constraint terms.

The widely used notation [15, 16] is to use the variables $\left(\phi, \tilde{\gamma}_{i j}, K, \tilde{A}_{i j}, \tilde{\Gamma}^{i}\right)$ instead of the standard ADM $\left(\gamma_{i j}, K_{i j}\right)$, where

$$
\begin{aligned}
\tilde{\gamma}_{i j} & =e^{-4 \phi} \gamma_{i j}, \\
\tilde{A}_{i j} & =e^{-4 \phi}\left(K_{i j}-(1 / 3) \gamma_{i j} K\right), \\
\tilde{\Gamma}^{i} & =\tilde{\Gamma}_{j k}^{i} \tilde{\gamma}^{j k}
\end{aligned}
$$

and we impose $\operatorname{det} \tilde{\gamma}_{i j}=1$ during the evolutions. The set of evolution equations become

$$
\begin{aligned}
\left(\partial_{t}-\mathcal{L}_{\beta}\right) \phi= & (-1 / 6) \alpha K \\
\left(\partial_{t}-\mathcal{L}_{\beta}\right) \tilde{\gamma}_{i j}= & -2 \alpha \tilde{A}_{i j}, \\
\left(\partial_{t}-\mathcal{L}_{\beta}\right) K= & \alpha\left(1-\kappa_{1}\right) R^{(3)}+\alpha\left(1-\kappa_{1}\right) K^{2}+\alpha \kappa_{1} \tilde{A}_{i j} \tilde{A}^{i j}+(1 / 3) \alpha \kappa_{1} K^{2}-\gamma^{i j}\left(\nabla_{i} \nabla_{j} \alpha\right), \\
\left(\partial_{t}-\mathcal{L}_{\beta}\right) \tilde{A}_{i j}= & -e^{-4 \phi}\left(\nabla_{i} \nabla_{j} \alpha\right)^{T F}+e^{-4 \phi} \alpha R_{i j}^{(3)}-e^{-4 \phi} \alpha(1 / 3) \gamma_{i j}\left(1-\kappa_{3}\right) R^{(3)}+\alpha\left(K \tilde{A}_{i j}-2 \tilde{A}_{i k} \tilde{A}^{k}{ }_{j}\right) \\
& +e^{-4 \phi} \alpha(1 / 3) \gamma_{i j} \kappa_{3}\left[-\tilde{A}_{k l} \tilde{A}^{k l}+(2 / 3) K^{2}\right], \\
\partial_{t} \tilde{\Gamma}^{i}= & -2\left(\partial_{j} \alpha\right) \tilde{A}^{i j}-(4 / 3) \kappa_{2} \alpha\left(\partial_{j} K\right) \tilde{\gamma}^{i j}+12 \kappa_{2} \alpha \tilde{A}^{j i}\left(\partial_{j} \phi\right)-2 \alpha \tilde{A}_{k}{ }^{j}\left(\partial_{j} \tilde{\gamma}^{i k}\right)-2 \kappa_{2} \alpha \tilde{\Gamma}^{k}{ }_{l j} \tilde{A}^{j}{ }_{k} \tilde{\gamma}^{i l} \\
& -2\left(1-\kappa_{2}\right) \alpha\left(\partial_{j} \tilde{A}_{k l}\right) \tilde{\gamma}^{i k} \tilde{\gamma}^{j l}+2 \alpha\left(1-\kappa_{2}\right) \tilde{A}_{j}^{i} \tilde{\Gamma}^{j} \\
& -\partial_{j}\left(\beta^{k} \partial_{k} \tilde{\gamma}^{i j}-\tilde{\gamma}^{k j}\left(\partial_{k} \beta^{i}\right)-\tilde{\gamma}^{k i}\left(\partial_{k} \beta^{j}\right)+(2 / 3) \tilde{\gamma}^{i j}\left(\partial_{k} \beta^{k}\right)\right)
\end{aligned}
$$


where $\mathcal{L}_{\beta}$ is the Lie derivative along the shift vector $\beta^{i}$, and $R^{(3)}$ is the 3 -metric scalar curvature. Here we introduced parameters $\kappa$ which show where we replace the terms with constraints. For example $\left(\kappa_{1}, \kappa_{2}, \kappa_{3}\right)=(0,0,0)$ is the case of no replacement (the standard ADM equations expressed using (5.1)-(5.3)), while Baumgarte-Shapiro [16] uses $\left(\kappa_{1}, \kappa_{2}, \kappa_{3}\right)=(1,1,0)$.

The constraint equations in CT-ADM system can be expressed as

$$
\begin{aligned}
\mathcal{H} & =e^{-4 \phi} \tilde{R}^{(3)}-8 e^{-4 \phi} \tilde{\gamma}^{i j}\left(\partial_{i} \partial_{j} \phi\right)-8 e^{-4 \phi} \tilde{\gamma}^{i j}\left(\partial_{i} \phi\right)\left(\partial_{j} \phi\right)+8 e^{-4 \phi}\left(\partial_{i} \phi\right) \tilde{\Gamma}^{i}+(2 / 3) K^{2}-\tilde{A}_{i j} \tilde{A}^{i j}, \\
\mathcal{M}_{i} & =\left(\partial_{j} \tilde{A}_{k i}\right) \tilde{\gamma}^{k j}-(2 / 3)\left(\partial_{i} K\right)-\tilde{A}_{j i} \tilde{\Gamma}^{j}+6\left(\partial_{j} \phi\right) \tilde{A}_{i}^{j}-\tilde{\Gamma}_{j i}^{k} \tilde{A}_{k}^{j}, \\
\mathcal{G}^{i} & =\tilde{\Gamma}^{i}+\partial_{j} \tilde{\gamma}^{j i}
\end{aligned}
$$

Here $\mathcal{H}, \mathcal{M}$ are the Hamiltonian and momentum constraints and the third one, $\mathcal{G}$, is a consistency relation due to the algebraic definition of (5.3).

\section{B. Constraint propagation equations of CT-ADM}

Similar to the ADM cases, we here show the propagation equations for (5.9)-(5.11). The expressions are given using (3.5) and (3.6), but we have to be careful to keep using the new variable, $\Gamma_{i}$, wherever it appears. Following [16], we express $\tilde{R}_{i j}^{(3)}$ as

$$
\begin{aligned}
\tilde{R}_{i j}^{(3)}= & -(1 / 2) \tilde{\gamma}^{l m}\left(\partial_{l} \partial_{m} \tilde{\gamma}_{i j}\right)+(1 / 2) \tilde{\gamma}_{k i} \partial_{j} \tilde{\Gamma}^{k}+(1 / 2) \tilde{\gamma}_{k j} \partial_{i} \tilde{\Gamma}^{k}+(1 / 2) \tilde{\Gamma}^{k} \tilde{\Gamma}_{(i j) k} \\
& +\tilde{\gamma}^{l m} \tilde{\Gamma}_{l i}^{k} \tilde{\Gamma}_{j k m}+\tilde{\gamma}^{l m} \tilde{\Gamma}_{l j}^{k} \tilde{\Gamma}_{i k m}+\tilde{\gamma}^{l m} \tilde{\Gamma}_{i m}^{k} \tilde{\Gamma}_{k l j} .
\end{aligned}
$$

The constraint propagation equations, then, are obtained by straightforward calculations as

$$
\begin{aligned}
\partial_{t} \mathcal{H}= & \beta^{j}\left(\partial_{j} \mathcal{H}\right)-2 \alpha e^{-4 \phi} \tilde{\gamma}^{i j}\left(\partial_{i} \mathcal{M}_{j}\right)+2 \alpha K \mathcal{H}-2 \alpha e^{-4 \phi}\left(\partial_{i} \tilde{\gamma}^{i j}\right) \mathcal{M}_{j}-4 \alpha e^{-4 \phi}\left(\partial_{i} \phi\right) \tilde{\gamma}^{i j} \mathcal{M}_{j}-4 e^{-4 \phi} \tilde{\gamma}^{i j}\left(\partial_{j} \alpha\right) \mathcal{M}_{i} \\
& +2 \kappa_{2} e^{-4 \phi}\left(\partial_{i} \alpha\right) \tilde{\gamma}^{i j} \mathcal{M}_{j}+2 \kappa_{2} e^{-4 \phi} \alpha\left(\partial_{i} \tilde{\gamma}^{i j}\right) \mathcal{M}_{j}+2 \kappa_{2} e^{-4 \phi} \alpha \tilde{\gamma}^{i j}\left(\partial_{i} \mathcal{M}_{j}\right) \\
& +16 \kappa_{2} \alpha e^{-4 \phi}\left(\partial_{i} \phi\right) \tilde{\gamma}^{i j} \mathcal{M}_{j}-(4 / 3) \kappa_{1} \alpha K \mathcal{H}, \\
\partial_{t} \mathcal{M}_{i}= & -(1 / 2) \alpha\left(\partial_{i} \mathcal{H}\right)+\beta^{j}\left(\partial_{j} \mathcal{M}_{i}\right)+\alpha K \mathcal{M}_{i}-\left(\partial_{i} \alpha\right) \mathcal{H}-4 \beta^{j}\left(\partial_{i} \phi\right) \mathcal{M}_{j}+\beta^{k} \tilde{\gamma}^{j l}\left(\partial_{i} \tilde{\gamma}_{l k}\right) \mathcal{M}_{j}+\left(\partial_{i} \beta_{k}\right) e^{-4 \phi} \tilde{\gamma}^{k j} \mathcal{M}_{j} \\
& +(1 / 3)\left(2 \kappa_{1}+\kappa_{3}\right)\left(\partial_{i} \alpha\right) \mathcal{H}+(1 / 3)\left(2 \kappa_{1}+\kappa_{3}\right) \alpha\left(\partial_{i} \mathcal{H}\right)-2 \kappa_{2} \alpha \tilde{A}^{j}{ }_{i} \mathcal{M}_{j}-(1 / 3) \kappa_{3} \alpha \mathcal{G}^{j} \tilde{\gamma}_{j i} \mathcal{H}+2 \kappa_{3} \alpha\left(\partial_{i} \phi\right) \mathcal{H}, \\
\partial_{t} \mathcal{G}^{i}= & 2 \tilde{A}^{i}{ }_{j} \mathcal{G}^{j}+2 \kappa_{2} \alpha \tilde{\gamma}^{i j} \mathcal{M}_{j} .
\end{aligned}
$$

These form a first order system, and the characteristic part can be extracted as

$$
\partial_{t}\left(\begin{array}{c}
\mathcal{H} \\
\mathcal{M}_{i} \\
\mathcal{G}^{i}
\end{array}\right) \cong\left(\begin{array}{ccc}
\beta^{l} & 2\left(-1+\kappa_{2}\right) \alpha \gamma^{l j} & 0 \\
\left((2 / 3) \kappa_{1}+(1 / 3) \kappa_{3}-(1 / 2)\right) \alpha \delta_{i}^{l} & \beta^{l} \delta_{i}^{j} & 0 \\
0 & 0 & 0
\end{array}\right) \partial_{l}\left(\begin{array}{c}
\mathcal{H} \\
\mathcal{M}_{j} \\
\mathcal{G}^{j}
\end{array}\right)
$$

whose characteristic speeds are

$$
\lambda^{l}=\left(0,0,0, \beta^{l}, \beta^{l}, \beta^{l} \pm \alpha \sqrt{\gamma^{l l}\left(1-\kappa_{2}\right)\left(1-(4 / 3) \kappa_{1}-(2 / 3) \kappa_{3}\right)}\right) \quad \text { (no sum over } l \text { ). }
$$

By analyzing the reality of the eigenvalues, the diagonalizability of the characteristic matrix, and the possibility of the symmetric characteristic matrix, we can classify the hyperbolicity of the system (5.16) as

$$
\begin{aligned}
\text { weakly hyperbolic } \Leftrightarrow & \left(1-\kappa_{2}\right)\left(1-(4 / 3) \kappa_{1}-(2 / 3) \kappa_{3}\right) \geq 0, \\
\text { strongly hyperbolic } \Leftrightarrow & \left(1-\kappa_{2}\right)=\left(1-(4 / 3) \kappa_{1}-(2 / 3) \kappa_{3}\right)=0, \\
& \text { or }\left(1-\kappa_{2}\right)\left(1-(4 / 3) \kappa_{1}-(2 / 3) \kappa_{3}\right)>0, \\
\text { symmetric hyperbolic } \Leftrightarrow & \left(-1+\kappa_{2}\right)=\left(1-(4 / 3) \kappa_{1}-(2 / 3) \kappa_{3}\right) .
\end{aligned}
$$

That is, for the non-adjusted system, $\left(\kappa_{1}, \kappa_{2}, \kappa_{3}\right)=(0,0,0)$, constraint propagation forms a strongly hyperbolic system, while the Baumgarte-Shapiro form gives only weakly hyperbolicity. (We note that the first-order version of CT-ADM by Frittelli-Reula [20] has also well-posed constraint propagation equations. ) 


\section{Amplification factors on Minkowskii background}

For a Minkowskii background, the constraint propagation equations at the linear order become

$$
\partial_{t}\left(\begin{array}{c}
(1) \hat{\mathcal{H}} \\
{ }^{(1)} \hat{\mathcal{M}}_{i} \\
{ }^{(1)} \hat{\mathcal{G}}^{i}
\end{array}\right)=\left(\begin{array}{ccc}
0 & 2\left(\kappa_{2}-1\right) i k_{j} & 0 \\
\left((2 / 3) \kappa_{1}+(1 / 3) \kappa_{3}-(1 / 2)\right) i k_{i} & 0 & 0 \\
0 & 2 \kappa_{2} \delta^{i j} & 0
\end{array}\right)\left(\begin{array}{c}
(1) \hat{\mathcal{H}} \\
(1) \hat{\mathcal{M}}_{i} \\
(1) \hat{\mathcal{G}}^{i}
\end{array}\right)
$$

The constraint amplification factor becomes

$$
\Lambda^{l}=\left(0,0,0,0,0, \pm \sqrt{-k^{2}\left(1-\kappa_{2}\right)\left(1-(4 / 3) \kappa_{1}-(2 / 3) \kappa_{3}\right)}\right)
$$

That is, $\Lambda^{l}$ are either zero, pure imaginary or \pm real numbers. For the non-adjusted system they are zero and pure imaginary (that is, the same as (3.11)), while the Baumgarte-Shapiro form gives us all zero eigenvalues. Therefore from our point of view, these two are not very different in their characterization of constraint propagation.

\section{CONCLUDING REMARKS}

We have reviewed ADM systems from the point of view of adjustment of the dynamical equations by constraint terms. We have shown that characteristic speeds and amplification factors of the constraint propagation change due to their adjustments. We compared the equations for the ADM, adjusted ADM, conformal traceless ADM (CT$\mathrm{ADM}$ ) systems, and tried to find the system that is robust for violation of the constraints, which we can call an "asymptotically constrained" system.

We conjectured that if the amplification factors (eigenvalues of the coefficient matrix of the Fourier-transformed constraint propagation equations) are negative or pure-imaginary, then the system has better asymptotically constrained features than a system they are not. According to our conjecture, the standard ADM system is expected to have better stability than the original ADM system (no growing mode in amplification factors). Detweiler's modified ADM system, which is one particular choice of adjustment, definitely has good properties in that there are no growing modes in amplification factors. We also showed that this can be obtained by a simpler choice of adjustment multipliers.

We also studied the CT-ADM system which is popular with numerical relativists nowadays. However, from our point of view, we do not see any particular advantages for CT-ADM system over the standard ADM system.

The reader might ask why we can break the time-reversal invariant feature of the evolution equations by a particular choice of adjusting multipliers against the fact that the "Einstein equations" are time-reversal invariant. This question can be answered by the following. If we take a time-reversal transformation $\left(\partial_{t} \rightarrow-\partial_{t}\right)$, the Hamiltonian constraint and the evolution equations of $K_{i j}$ keep their signatures, while the momentum constraints and the evolution equations of $\gamma_{i j}$ change their signatures. Therefore if we adjust $\gamma_{i j}$-equations using Hamiltonian constraint and/or $K_{i j}$-equations using momentum constraints (supposing the multiplier has +-parity), then we can break the time-reversal invariant feature of the "ADM equations". In fact, the examples we obtained all obey this rule. The CT-ADM formulation keeps its signature against the adjustments we made, so that we can not find any additional advantage from this analysis.

Considering the constraint propagation equations is a kind of substitutional approach for numerical integrations of the dynamical equations. However, this might be one of the main directions for our future research, as Friedrich and Nagy [25] impose the zero speed of the constraint propagation as the first principle when they considered the initial boundary value problem of the Einstein equations [26].

We are now applying our discussion to more general spacetimes, and trying to find guidelines for choosing appropriate gauge conditions from the analysis of the constraint propagation equations. These efforts will be reported elsewhere [27].

\section{ACKNOWLEDGEMENTS}

HS appreciates helpful comments by Pablo Laguna, Jorge Pullin, Manuel Tiglio and the hospitality of the CGPG group. We also thank communication with Steven Detweiler. We thank Bernard Kelly for careful reading of the manuscript. This work was supported in part by the NSF grant PHY00-90091, and the Everly research funds of Penn State. HS was supported by the Japan Society for the Promotion of Science as a research fellow abroad. 


\section{APPENDIX A: NUMERICAL DEMONSTRATIONS OF ADJUSTED-ADM SYSTEMS}

We here show two numerical demonstrations of adjusted-ADM systems that were discussed in $\S$ IVC (Detweiler's modified ADM system) and $\S$ IV D 1 (simplified version).

Detweiler's adjustment, (4.12)-(4.14), can be parametrized by a constant $L$, and our prediction from the amplification factor on Minkowskii background is that this system will be asymptotically constrained for small positive $L$. Fig.11 is a demonstration of this system. We evolved Minkowskii spacetime numerically in a plane-symmetric spacetime, and added artificial error in the middle of the evolution. Our numerical integration uses the Brailovskaya scheme, which was described in detail in our previous paper [6]. The code passes convergence tests and the plots are for 401 gridpoints in the range $x=[0,10]$, and we fix the time grid $\Delta t=0.2 \Delta x$. The error was introduced as a pinpoint kick, in the form of $\Delta g_{y y}=10^{-3}$ at $x=5.0$ and $t=0.25$. We monitor how the L2 norm of the constraints $\left(\mathcal{H}^{2}+\mathcal{M}_{x}^{2}\right)$ behaves. From Fig. 1], we see that a small positive $L$ reduces the L2 norm in time, which is the asymptotically constrained feature we expected. The case of slightly larger $L$ will make the system unstable. This is the same feature we have seen in the numerical demonstration of the $\lambda$-system or adjusted-Maxwell/Ashtekar systems [11], for that case the upper bound of the multiplier can be explained by violation of the Courant-Friedrich-Lewy condition, while in this system we can not calculate the exact characteristics since the system is not first-order.

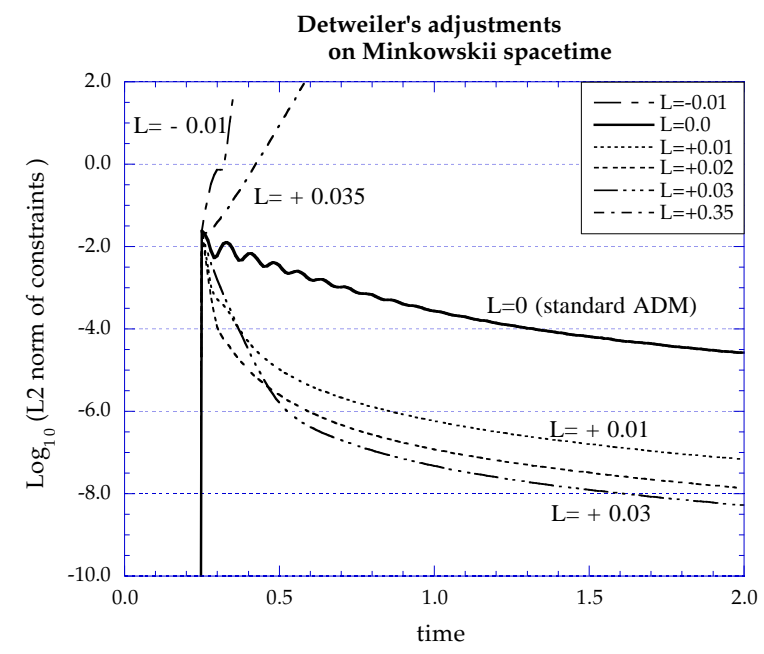

FIG. 1. Demonstration of the Detweiler's modified ADM system on Minkowskii background spacetime (the system of ¿V ). The L2 norm of the constraints is plotted in the function of time. Artificial error was added at $t=0.25$. $L$ is the parameter used in 4.12)-(4.14). We see the evolution is asymptotically constrained for small $L>0$.

Similarly, we plotted in Fig. 2 the case of simplified version (the system of $\S$ [V D 1). We see the desired feature again by changing the parameter $\kappa_{0}$ that appear in (4.22). 


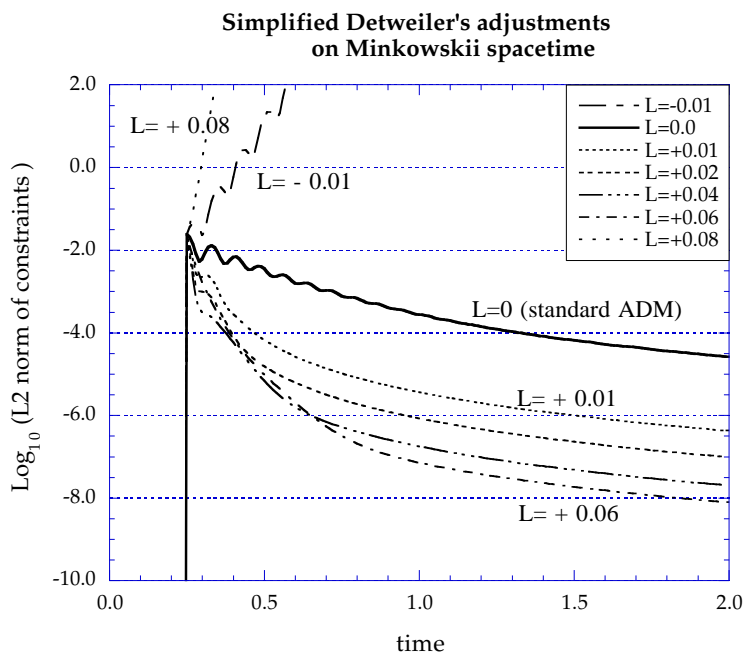

FIG. 2. Demonstration of the simplified Detweiler's modified ADM system on Minkowskii background spacetime (the system of $\mathrm{IVD}_{\mathrm{I}}$ ). For comparison with Fig.1, we set $L=-\kappa_{0}$, where $\kappa_{0}$ is the parameter used in (4.22). We see the evolution is asymptotically constrained for small $L>0$.

[1] R. Arnowitt, S. Deser and C.W. Misner, "The Dynamics of General Relativity", in Gravitation: An Introduction to Current Research, ed. by L.Witten, (Wiley, New York,1962).

[2] See the references in 6].

[3] G. Yoneda and H. Shinkai, Phys. Rev. Lett. 82, 263 (1999).

[4] G. Yoneda and H. Shinkai, Int. J. Mod. Phys. D. 9, 13 (2000).

[5] A. Ashtekar, Phys. Rev. Lett. 57, 2244 (1986); Phys. Rev. D36, 1587 (1987); Lectures on Non-Perturbative Canonical Gravity (World Scientific, Singapore, 1991).

[6] H. Shinkai and G. Yoneda, Class. Quant. Grav. 17, 4799 (2000).

[7] S. D. Hern, PhD thesis, gr-qc/0004036.

[8] S. Frittelli and O.A. Reula, Phys. Rev. Lett. 76, 4667 (1996). See also J. M. Stewart, Class. Quant. Grav. 15, 2865 (1998).

[9] O. Brodbeck, S. Frittelli, P. Hübner, and O.A. Reula, J. Math. Phys. 40, 909 (1999)

[10] H. Shinkai and G. Yoneda, Phys. Rev. D60, 101502 (1999)

[11] G. Yoneda and H. Shinkai, Class. Quant. Grav. 18, 441 (2001).

[12] J.W. York, Jr., "Kinematics and Dynamics of General Relativity", in Sources of Gravitational Radiation, ed. by L.Smarr, (Cambridge, 1979) ; L. Smarr, J.W. York,Jr., Phys. Rev. D17, 2529 (1978).

[13] S. Frittelli, Phys. Rev. D55, 5992 (1997).

[14] S. Detweiler, Phys. Rev. D35, 1095 (1987).

[15] T. Nakamura and K. Oohara, in Frontiers in Numerical Relativity edited by C.R. Evans, L.S. Finn, and D.W. Hobill (Cambridge Univ. Press, Cambridge, England, 1989). M. Shibata and T. Nakamura, Phys. Rev. D52, 5428 (1995).

[16] T. W. Baumgarte and S. L. Shapiro, Phys. Rev. D59, 024007 (1999).

[17] M. Alcubierre, B. Brügmann, T. Dramlitsch, J. A. Font, P. Papadopoulos, E. Seidel, N. Stergioulas, and R. Takahashi, Phys.Rev. D62, 044034 (2000).

[18] L. Lehner, M. Huq, D. Garrison, Phys. Rev. D62, 084016 (2000).

[19] A. Arbona, C. Bona, J. Massó, and J. Stela, Phys. Rev. D60, 104014 (1999).

[20] S. Frittelli and O. A. Reula, J. Math. Phys. 40, 5143 (1999).

[21] M. Alcubierre, B. Brügmann, M. Miller and W-M. Suen, Phys. Rev. D60, 064017 (1999).

[22] M. Alcubierre, G. Allen, B. Brügmann, E. Seidel and W-M. Suen, Phys. Rev. D62, 124011 (2000).

[23] M. Miller, gr-qc/0008017.

[24] S. Frittelli and R. Gomez, J. Math. Phys. 41, 5535 (2000).

[25] H. Friedrich and G. Nagy, Comm. Math. Phys. 201, 619 (1999).

[26] We thank M. Tiglio for pointing out this.

[27] H. Shinkai and G. Yoneda, in preparation. 